\title{
Structural, Vibrational and Thermal Properties of Semi Organic Nonlinear Optical Glutamic Added Lithium Sulphate Monohydrate Crystal
}

\author{
G. Theophil Anand*, S. Rama \\ Department of Physics and Abraham Panampara Research Centre, Sacred Heart College (Autonomous), Tirupattur - 635 601, Tamil Nadu, India.
}

\section{ARTICLE DETAILS}

\section{Article history:}

Received 24 February 2018

Accepted 22 March 2018

Available online 02 April 2018

\section{Keywords:}

Optical Studies

Mechanical Studies

NLO Study

\begin{abstract}
A B S T R A C T
Glutamic acid lithium sulphate monohydrate crystal is a new semiorganic nonlinear optical material. It was grown from a mixture of aqueous solution of glutamic acid and lithium sulphate monohydrate by slow evaporation technique at room temperature. The powder XRD analysis was carried out to identify the structural parameters of the grown crystals. The presence of functional groups of both reactants was qualitatively analyzed by means of Fourier transform infrared (FT-IR) spectrometer study. In UV-vis study, the existence of wide transparency window and band gap was investigated for optoelectronic device applications. In Kurtz-Perry powder technique, the second harmonic generation (SHG) efficiency of GLSMH crystal is found to be 1.933 times greater than that of KDP crystal.
\end{abstract}

\section{Introduction}

The non-linear optical (NLO) materials are at the fore front of the researches due to their wider applications in photonics and optical communication devices. Owing to the increasing need of new materials with high NLO efficiency and improved physical properties, new approach has been developed to hybrid organic-inorganic materials with their advantages for technological applications [1]. The materials that have a potential to tailor high optical nonlinearity of an organic ion with favorable mechanical and thermal properties of an inorganic counter ion, turned out to be superior quality semi organic materials $[2,3]$. Extensive research has revealed that Amino acids serve as attractive organic candidates , as they contain the chiral nature, wide hydrogen bonding network, a proton donor carboxyl $(-\mathrm{COOH})$ group and a proton acceptor amine $\left(-\mathrm{NH}_{2}\right)$ group with a side chain (R) group specific to each amino acid. Complexes of amino acids with inorganic salts are suitable for optical device applications. Hence amino acids offer a wide range of choice to synthesize the new semi organic materials with large enhancement in properties of NLO crystals [4]. L-proline lithium bromide monohydrate [5], L-proline lithium chloride monohydrate [6], L-valine cadmium chloride monohydrate [7], L-valine ferric chloride [8], L-arginine hydrochloride bromide [9], L-arginine hydrobromide monohydrate [10] and L-threonine copper (II) monohydrate [11] are some of the amino acid based semi organic materials reported with enriched NLO efficiency and optical quality. Glutamic acid is a monoamino dicarboxylic $\alpha$-aminoacid and a significant constituent of proteins such as myosin, insulin and edestin. It was shown to be phase matchable for optical second harmonic generation of $1.06 \mu \mathrm{m}$ and has nonlinear optical co-efficient [12-13]. From the literature, it is inferred that L-glutamic acid has been proved to have improved NLO efficiency upon the reaction with inorganic hydrochloric acid [14] and hydrobromic acid. The combination of simplest amino acid glycine with lithium sulphate monohydrate exhibits high nonlinear optical property. However there is no report available so far on the compound of L-Glutamic acid with lithium sulphate monohydrate. The present work reports for the first time the synthesis, crystal growth and characterization of a new semi organic material, where the glutamic acid has been chosen to be the organic ion and Lithium sulphate monohydrate that has potential applications in technological devices, such as pyroelecrtric, nonlinear optical, and ferroelectric devices to be the inorganic counterpart in the present study [15-17]. The improved nonlinearity and physical properties are typical of the strategy targeted in engineering semi organic material for nonlinear optical device applications. Having gone through many articles regarding single crystal grown by slow evaporation method which has NLO properties, and especially the last four articles mentioned in the literature survey has given vigour to do a project in lithium sulphate monohydrate and glutamic acid. Besides, the synthesis of lithium sulphate monohydrate oxalate gives a clear idea about the semi-organic materials for NLO applications. Also it is obvious that lithium sulphate monohydrate (LSMH) is an inorganic material and has a NLO property and glutamic acid is an organic material and when both are combined together it forms as a semi-organic material for an enhanced NLO properties. Hence, the work focuses on the preparation of pure lithium suphate monohydrate (LSMH) and glutamic added lithium sulphate monohydrate (GLSMH) and comparison of the same for NLO applications.

\section{Experimental Methods}

\subsection{Materials}

The materials used are lithium sulphate monohydrate $\left(\mathrm{Li}_{2} \mathrm{SO}_{4} \cdot \mathrm{H}_{2} \mathrm{O}\right)$ (Merck) (99\% AR grade), glutamic acid (Merck) (99\% AR grade), deionized water and $\mathrm{NaOH}$ to adjust $\mathrm{pH}$.

\subsection{Synthesis of Crystal Growth}

Lithium sulphate monohydrate crystal was synthesized from glutamic acid and lithium sulphate monohydrate by taking in equimolar ratio (1:1). A calculated amount of the reactants was dissolved in deionized water and stirred well using magnetic stirrer and a homogeneous mixture was obtained. The solution was filtered and allowed for slow evaporation at room temperature. After a span of 15 days, a single nucleation started and the grown crystals were harvested over a period of 30 days. A calculated amount of the reactants was dissolved in deionized water and stirred well using magnetic stirrer and a homogeneous mixture was obtained. After that the $\mathrm{PH}$ of the saturated solution was adjusted to 10.4 , which is one unit greater than that of the Pka value using $\mathrm{NaOH}$ to stimulate the dissociation of the compound. The solution was filtered and allowed for slow evaporation at room temperature. After a span of 15 days, a single nucleation started and the grown crystals of the size $3 \times 2.5 \times 1 \mathrm{~mm}^{3}$ were harvested over a period of 30 days. The photograph of grown crystals is shown in Fig. 1. 


\subsection{Characterization of Pure and Glutamic Added LSMH}

Structural analysis were confirmed by X-Ray powder diffraction (XRD), the presence the vibrational modes were identified using Fourier transform infrared spectrometry (FT-IR), UV-visible spectrum confirm the absorption of grown crystal. The stability of the material was found by thermo gravimetric analysis (TGA) and differential thermal analysis (DTA) technique. The powder SHG technique confirms the presence second harmonic generation of the material.

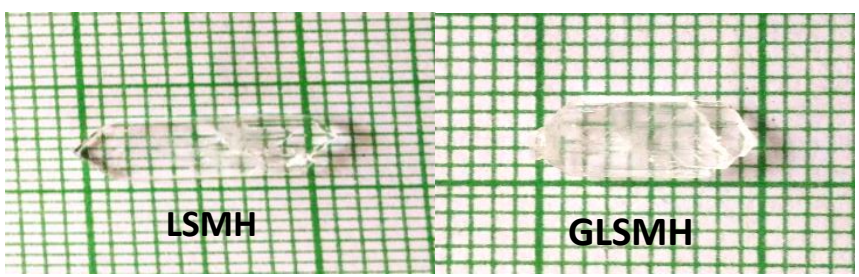

Fig. 1 The photograph of LSMH and GLSMH

\section{Results and Discussion}

\subsection{Structural Analysis}

The unit cell parameters and crystalline phase of the grown crystals have been determined by analyzing the powder XRD pattern of L-glutamic lithium sulphate monohydrate that was recorded by employing XPERTPRO diffractometer system with Cuk $\alpha$ radiation of wavelength $\lambda=1.54060$ $\AA$. The XRD pattern of L-GLSMH in Fig. 2 shows the sharp and major reflecting planes confirming crystalline purity. The analysis of powder XRD pattern was done using the powder X software. The indexed peaks in the pattern depict standard assigned peaks of lithium sulphate monohydrate along with few additional peaks. There is observed change in the intensity of faces 101 and 110 , compared with the standard XRD pattern of LSMH crystals. The powder XRD data revealed that the LGLSMH was crystallized in monoclinic structure having space group $\mathrm{P}_{21}$ with unit cell parameters: $a=17.8892 \AA, b=5.8714 \AA, c=9.6801 \AA$. The slight changes in the lattice parameters in reference to the pure LSMH and minute alteration in the 101 and 110 face intensities indicate the strain imposed by glutamic acid on the lattice site of the lithium sulphate monohydrate.

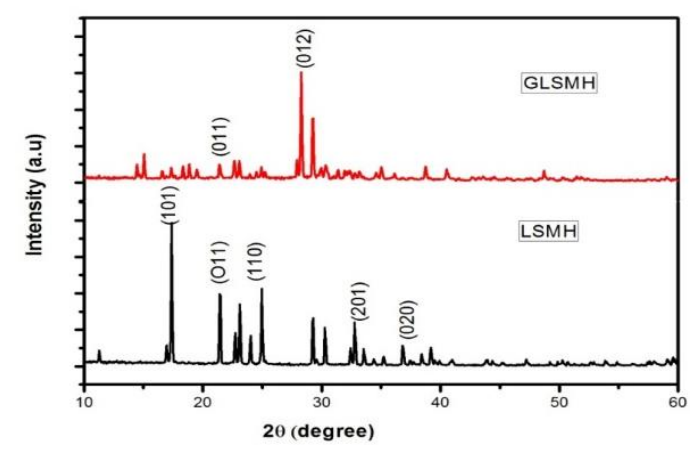

Fig. 2 Powder XRD pattern of LSMH \& GLSMH

\subsection{Functional Group Analysis}

The functional groups of the compound have been identified by using FTIR spectrum that was recorded for the fine powder of Lithium sulphate monohydrate in the wavelength region between $400-4000 \mathrm{~cm}^{-1}$ using Perkin Elmer Spectrum RX I spectrometer. The spectrum of LSMH is shown in Fig. 3. The assignments are discussed in three different regions: a high wave number region (4000-3000 $\left.\mathrm{cm}^{-1}\right)$, a functional group region (3000-1500 $\left.\mathrm{cm}^{-1}\right)$, and a fingerprint region $\left(1500-400 \mathrm{~cm}^{-1}\right)$ [18]. The broad peak observed at $3499 \mathrm{~cm}^{-1}$ is attributed to $\mathrm{O}-\mathrm{H}$ stretch vibration of lithium sulphate monohydrate. The characteristic stretching vibrations of SO ${ }^{4-}$ group appear at 1111 and $638 \mathrm{~cm}^{-1}$, which are in good agreement with compounds containing lithium sulphate monohydrate [19].

The functional groups of the compound have been identified by using FTIR spectrum that was recorded for the fine powder of L-glutamic lithium sulphate monohydrate in the wavelength region between $400-4000 \mathrm{~cm}^{-1}$ using Perkin Elmer Spectrum RX I spectrometer. The spectrum of LGLSMH is shown in Fig. 3. The assignments are discussed in three different regions: a high wave number region (4000-3000 $\left.\mathrm{cm}^{-1}\right)$, a functional group region $\left(3000-1500 \mathrm{~cm}^{-1}\right)$, and a fingerprint region $\left(1500-400 \mathrm{~cm}^{-1}\right)$. The broad peak observed at $3497 \mathrm{~cm}^{-1}$ is attributed to $\mathrm{O}-\mathrm{H}$ stretch vibration of https://doi.org/10.30799/jnst.102.18040110 lithium sulphate monohydrate. The strong peaks at 1723 and $1761 \mathrm{~cm}^{-1}$ are assigned to $\mathrm{C}=0$ stretch. This is slightly shifted due the compound formation. The $\mathrm{NH}_{3}{ }^{+}$symmetric deformation is observed at $1514 \mathrm{~cm}^{-1}$. The characteristic stretching vibrations of $\mathrm{SO}_{4}{ }^{2-}$ group appear at 1115 and 638 $\mathrm{cm}^{-1}[18,19]$, which are in good agreement with compounds containing lithium sulphate monohydrate.
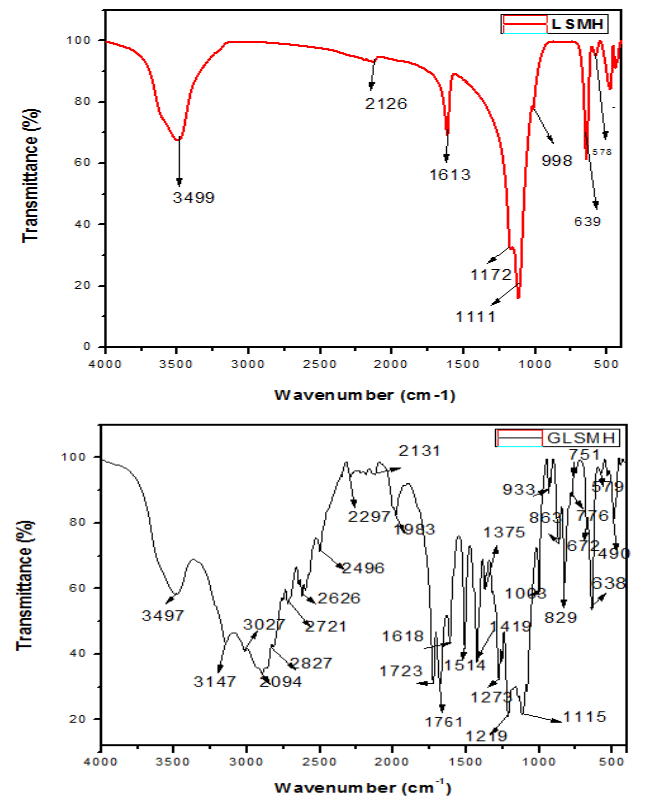

Fig. 3 FTIR spectrum of LSMH and GLSMH

\subsection{Linear Optical Analysis}

The optical transmission range, transparency cut-off and absorbance band are important optical parameters for laser frequency conversion applications [20]. Hence, the estimation of optical transmission range of the grown crystal for NLO applications becomes significant. The optical transmission spectra of lithium sulphate monohydrate crystal were recorded using Perkin Elmer Lambda 35, a high resolution UV Visible spectrophotometer in the wavelength region $190 \mathrm{~nm}$ to $1100 \mathrm{~nm}$ and the resulting spectrum is given in Fig. 4. The grown crystal has lower cut off wavelength $226 \mathrm{~nm}$ along with sufficient transmission in the entire visible region. This makes it a potential material for blue light emission. The optical band gap (Eg) evaluated is $5.49 \mathrm{eV}$. The existence of wide transparency in LSMH crystal offers reduced scattering and absorption of light in the visible region owing to the intrinsic property of the counter ion (amino acid), and minimized defects (structural and crystalline). This enforces substantial enrichment of optical quality of the grown GLSMH crystals. The good optical transparency observed in GLSMH crystal pronounces it as a suitable material for the transmission of SHG radiation of Nd:YAG laser ,in designing NLO devices and UV tunable lasers.

The optical transmission spectra of glutamic lithium sulphate monohydrate crystal were recorded using Perkin Elmer Lambda 35, a high resolution UV Visible spectrophotometer in the wavelength region $190 \mathrm{~nm}$ to $1100 \mathrm{~nm}$ and the resulting spectrum is given in Fig. 4. The grown crystal has lower cut off wavelength $256 \mathrm{~nm}$ along with sufficient transmission in the entire visible region. This makes it a potential material for blue light emission $[19,20]$. The optical band gap (Eg) evaluated is $4.85 \mathrm{eV}$.The existence of wide transparency in L-GLSMH crystal offers reduced scattering and absorption of light in the visible region owing to the intrinsic property of the counter ion (amino acid), and minimized defects (structural and crystalline). This enforces substantial enrichment of optical quality of the grown L-GLSMH crystals. The good optical transparency observed in L-GLSMH crystal pronounces it as a suitable material for the transmission of SHG radiation of Nd:YAG laser in designing NLO devices and UV tunable lasers [20].
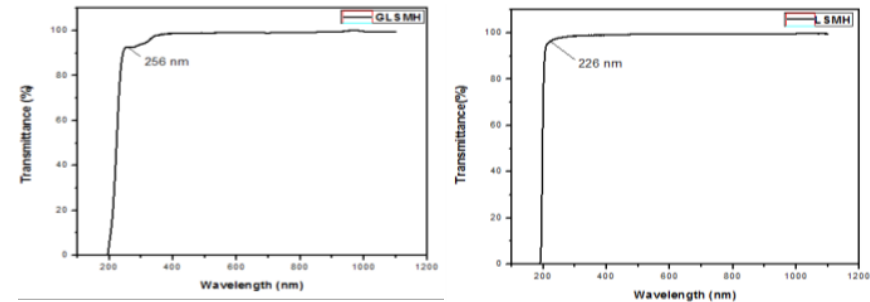

Fig. 4 UV-Visible spectrum of LSMH and GLSMH 


\subsection{TGA and DTA Analysis}

The TGA and DTA curve of LSMH crystals were shownin Fig. 5. The water molecules will be evaporated at the temperature of $103{ }^{\circ} \mathrm{C}$ of LSMH. The phase transition takes place from $103^{\circ} \mathrm{C}$ upto $127^{\circ} \mathrm{C}$. from the DTA curve, there is one exothermic and one endothermic peaks were observed. The TGA and DTA curve of GLSMH crystals were shownin Fig. 5. The water molecules will be evaporated at the temperature of $206^{\circ} \mathrm{C}$ of GLSMH. The phase transition takes place from $103{ }^{\circ} \mathrm{C}$ upto $243^{\circ} \mathrm{C}$. from the DTA curve, there is one exothermic and one endothermic peaks were observed.

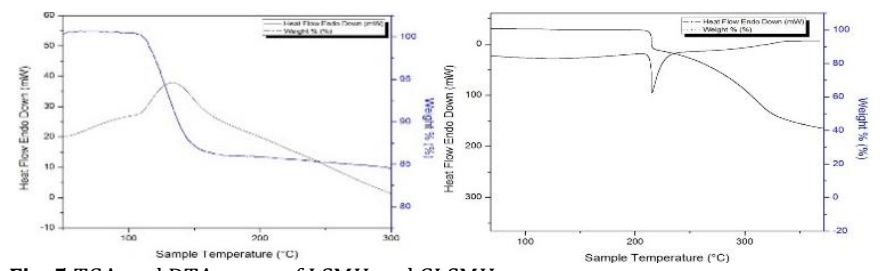

Fig. 5 TGA and DTA curve of LSMH and GLSMH

\subsection{Nonlinear Optical Analysis (SHG Measurement)}

The second harmonic generation efficiency of the crystal was studied by the powder technique developed by Kurtz and Perry [21]. The powdered sample of the grown crystal was packed into quartz capillary tube. After that, the sample was radiated by the beam of wavelength $1064 \mathrm{~nm}$ from Nd: YAG laser source. The output indicates that the SHG efficiency of LGLSM is 1.933 times greater than that of KDP. This enhanced NLO coefficient is another important expected outcome of semiorganic crystal formation.

\section{Conclusion}

New semi organic good quality L-glutamic lithium sulphate monohydrate crystals were grown from solution by slow evaporation technique. The structure of the grown crystal was found to be monoclinic with space group $\mathrm{P} 2{ }_{1}$ and the reflecting planes were indexed. The cut off wavelength and optical band gap value signify that this material possesses enhanced optical quality. The functional group identification communicate that this grown crystal is a hybrid of organic (L-glutamic acid) ion with inorganic (lithium sulphate monohydrate) ion. The improved work hardening coefficeient and nonlinear optical efficiency are the achievements of engineering semiorganic material. Wide optical transparency in the visible region, improved work hardening coefficients and enhanced NLO properties are highly favourable parameters for NLO device fabrication.

\section{References}

[1] Tanusri Pal, Tanusree Kar, Gabriele Bocelli, Lara Rigi, Synthesis, growth and characterization of L-arginine acetate crystal: A potential NLO material, Cryst. Growth Design. 3(1) (2003) 13-16.
[2] Md. Shkir, S. Alfaify, M. Ajmal Khan, E. Dieguez, J. Perles, Synthesis, growth crystal structure, EDX, UV-Vis-NIR and DSC studies of L-proline lithium bromide monohydrate- A new semi organic compound, J. Cryst. Growth 391 (2014) 104-110.

[3] S. Sathiskumar, T. Balakrishnan, K. Ramamurthi, S. Thamotharan, Synthesis structure, crystal growth and characterization of novel semiorganic nonlinear optical L-proline bromide monohydrate single crystals, Spectrochim. Acta A 138 (2014) 1386-1425.

[4] T. Uma Devi, N. Lawrence, R. Ramesh Babu, S. Selvanayagam, Helen SteockliEvans, K. Ramamurthi, Synthesis, crystal growth and characterization of Lproline chloride monohydrate: A New semi organic nonlinear optical material, Cryst. Growth Design 9 (2009) 1370-1374.

[5] P. Maadeswaran, J. Chandrasekaran, Synthesis, growth and characterization of L-valine cadmium chloride monohydrate- A novel semiorganic nonlinear optical crystal, Optik 122 (2011) 1128-1131.

[6] P. Geetha, S. Krishnan, R.K. Natarajan, V. Chithambaram, Growth and characterization of semiorganic nonlinear optical L-valine ferric chloride single crystal by solution growth technique, Curr. Appl. Phy. 15 (2015) 201-207.

[7] T. Pal, Tanusree Kar, G. Bocelli, Lara Rigi, Synthesis, crystal structure and thermal and spectral studies of semiorganic nonlinear optical Crystal $\mathrm{LAHClBr}$ Cyst. Growth Design 4 (2004) 743-747.

[8] S. Mukerji, T. Kar, Structural, thermal, spectroscopic investigation of nonlinear optical crystal L-arginine hydrobromide monohydrate, Mat. Res. Bull. 33 (1998) 619-626.

[9] R. Subhashini, D. Sathya, V. Sivashankar, P.S. Latha Mageshwari, S. Arjunan, Growth and characterization of bis(L-threonine) copper (II) monohydrate single crystals: A semiorganic second order nonlinear optical material, Optic. Mater. 62 (2016) 357-365.

[10] M. Delfino, J.P. Dougherty, W.K. Zwicker, M.M. Choy, Solution growth and characterization of $\mathrm{L}(+)$ glutamic acid hydrochloride single crystals, J. Cryst. Growth 36 (1976) 267-272.

[11] M. Delfino, G.M. Loiacono, J. Fitzpatrick, W.A. Smith, Deuterium isotope effect in L (+) glutamic acid hydrochloride, J. Solid State Chem. 20 (1977) 391-395.

[12] J. Uma, V. Rajendran, Growth and properties of semiorganic nonlinear optical crystal: L-Glutamic acid hydrochloride, Prog. Natural Sci. Mater. Int. 26(1) (2016) 1-8.

[13] R. Sathyalakshmi, G. Bhagavannarayana, P. Ramasamy, Growth and characterization of a new NLO material: L- glutamic acid hydro bromide (LGluHBr), Mat. Res. Bull. 44 (2009) 1097-1101.

[14] T. Balakrishnan, K. Ramamurthi, Growth and characterization of glysine lithium sulphate single crystal, Cryst. Res. Technol. 41 (2006) 1184-1188.

[15] L. Bayajargal, Nonlinear optical properties of lithium sulfate monohydrate, Cryst. Res. Technol. 43 (2008) 1138-1142.

[16] K. Boopathi, R. Ramasamy, G. Bhagavannarayana, Growth and characterization of $\mathrm{Cu}(\mathrm{II})$ doped negatively soluble lithium sulphate monohydrate crystals, J. Cryst. Growth 386 (2014) 32-37.

[17] G. Emerson Robin, U. Sankar, T. Chithambarathanu, P. Selvarajan, Structural, thermal, impedance, FTIR and EDAX studies of lithium formate monohydrate crystals grown by solution method, Int. J. Innovat. Res. Adv. Eng. 2(6) (2015) 2349-2163.

[18] R. Bairava Ganesh, V. Kannan, R. Sathyalakshmi, P. Ramasamy, The growth of L-glutamic acid hydrochloride crystals by Sankaranarayanana-Ramasamy(SR) method, Mater. Lett. 61 (2007) 706-708.

[19] K. Ambujam, S. Selvakumar, D. Prem Anand, G. Mohamed, P. Sagayaraj, Crystal growth, optical, mechanical and electrical properties of organic NLO material $\gamma$-glycine, Cryst. Res. Technol. 41(7) (2006) 671-677.

[20] Mohd Anis, G.G. Muley, V.G. Pahurkar, M.I. Baig, S.R. Dagdale, Influence of $\mathrm{Nd}^{3+}$ on zinc tris-thiourae sulphate single crystal: a comparative crystal growth, structural, linear-nonlinear optical and dielectric study to explore NLO device applications, Mater. Res. Innovat. 22(2) (2016) 99-106.

[21] S.K. Kurtz, T.T. Perry, A Powder technique for the evaluation of nonlinear optical materials, J. Appl. Phys. 39 (1968) 3798-3813. 